\title{
A Hospital Based Study in South Indian Population on the Role of Ultrasound in the Estimation of Gestational Age in the Third Trimester of Pregnancy
}

\author{
Farhana Aljabri ${ }^{1}$, Sharmila ${ }^{2}$, K.S. Saraswathi ${ }^{1}$ and R. Shyamala ${ }^{3^{*}}$ \\ ${ }^{1}$ Department of $O B G Y$, Shadan Institute of Medical Sciences and P.G Research Centre, \\ Hyderabad, Telangana State, India \\ ${ }^{2}$ Department of OBGY, Gandhi Medical College, Hyderabad, Telangana State, India \\ ${ }^{3}$ Department of Microbiology, Malla Reddy Medical College for Women, Hyderabad, \\ Telangana State, India \\ *Corresponding author
}

\begin{abstract}
A B S T R A C T
Gestational age, synonymous with menstrual age, is defined in weeks beginning from the day of the last menstrual period prior to conception. Correct determination of gestational

\section{Keywords}

Gestational age,

Ultrasonography,

Crown rump length,

Bi-parietal

Diameter

Article Info

Accepted:

12 February 2018

Available Online:

10 March 2018

age is basic to obstetric care as it is very important in a number of circumstances. The study was undertaken at Shadan Institute of Medical Sciences and Post Graduate Research Centre, Hyderabad from June 2011 to December 2012. The study was a prospective study which included one hundred and fifty pregnant women who attended the antenatal outpatient of the Obstetrics Department. They were subjected to a single Sonographic examination in the III trimester and the gestational age was estimated using different foetal growth parameters like Biparietal diameter, femur length and abdominal circumference. Next the two parameters viz., BPD and FL were compared and their accuracy in estimation of gestational age was studied. One hundred and fifty patients, who fulfilled the subject selection criteria were included in this study. In the present study 72 cases $(48 \%)$ were primis, 50 cases $(33.33 \%)$ were gravid II and 28 cases $(18.67 \%)$ were gravid III and above. In our country where most of the women many don't keep menstrual record properly and who may present late in third trimester for the first time, ultra-sonographically assessed gestational age by multiple foetal growth parameters can be of immense value.
\end{abstract}

\section{Introduction}

Gestational age, synonymous with menstrual age, is defined in weeks beginning from the day of the last menstrual period prior to conception. Correct determination of gestational age is basic to obstetric care as it is very important in a number of circumstances. The level of serum alpha- fetoprotein in both maternal serum and amniotic fluids is related to gestational age and when the data are inaccurate, test result will be misleading and incorrect.

Timing of repeat caesarean section requires accurate assessment of dates and ultrasound is a reliable method for determining the length of pregnancy. Obstetric management is 
dependent on gestational age for e.g., a correct decision regarding preterm labour or postdated pregnancies are possible only when gestational age is correctly estimated.

Ultrasound has become the essential tool of modern Obstetric practice. With advances in technology and computer processing what was once a mere curiosity has become crucial for the assessment of placenta, membranes amniotic fluid and foetal anatomy.

Ultrasound technology gives insight into the intrauterine environment and makes it possible to monitor the development of embryo and foetus from the early pregnancy. Obstetric clinician sees various conditions of low incidence that may become clinically manifest only very late in pregnancy.

\section{Materials and Methods}

The study was undertaken at Shadan Institute of Medical Sciences and Post Graduate Research Centre, Hyderabad from June 2011 to December 2012.

The study was a prospective study which included one hundred and fifty pregnant women who attended the antenatal outpatient of the Obstetrics Department. They were subjected to a single Sonographic examination in the III trimester and the gestational age was estimated using different foetal growth parameters like Biparietal diameter, femur length and abdominal circumference.

Next the two parameters viz., BPD and FL were compared and their accuracy in estimation of gestational age was studied.

One hundred and fifty patients, who fulfilled the subject selection criteria were included in this study.

The selection criteria are as follows:-
The pregnant woman with previous regular menstrual cycles.

She knows the last menstrual period correctly.

She has not used any oral contraceptive pill, three months prior to pregnancy.

Height of the woman average.

Singleton pregnancies and vertex presentation.

\section{Exclusion criteria}

Exclusion criteria included any condition which affects the normal growth of the foetus. They are as follows:

Material Conditions:

Hypertension

Diabetes Mellitus

Heart Diseases

Tuberculosis

Anaemia

Multiple pregnancies

Pregnancy induce hypertension

Malpresentation

Poly hydramnios or oligohydramnios

Intra Uterine Growth Retardation

\section{Foetal conditions}

Anencephaly

Hydrocephalus

Foetal dwarfism

Foetal ascites

These one hundred and fifty women included in the study were explained regarding the study and their consent taken.

Prior approval of the study was taken from the ethical committee of Shadan Institute of Medical sciences and Post Graduate research Centre. 
Firstly, a thorough history was taken including the Obstetric history, menstrual history, and post history.

The weight, height of the patients recorded their B.P recorded. A complete obstetric examination was carried out. Preliminary investigations like $\mathrm{Hb} \%$, Urine exam, and other routine tests were carried out.

After this, these women were subjected to ultrasound examination for estimation of gestational age, using BPD, femur length, and abdominal circumference as standard parameters.

A real time 2-D ultrasound unit with a 3.5 $\mathrm{MHz}$ convex sector, transducer sonoline SL grey scale model with $\mathrm{M}$ and $\mathrm{B}$ mode for simultaneous imaging and calculating the foetal heart rate was used. After initial survey with the ultrasound transducer, the parameters obtained were: Biparietal diameter, femur length, abdominal circumference and foetal weight.

\section{First trimester assessment}

In the first trimester, gestational sac mean diameter and crown-rump length are used to establish foetal age. Both parameters are useful because each measures a different aspect of the first trimester pregnancy and may be used at different times during the first trimester.

\section{Gestational SAC diameter}

The gestational sac is the first identfiable structure routinely imaged in the first trimester. It is identified by transabdominal ultrasound as early as 5weeks gestational and may be seen as early as 4 weeks gestation by transvaginal ultrasound. The gestational sac is an echo-free space containing the fluid, embryo, and extra embryonic structures.

\section{Crown - Rump Length}

The Crown -rump length (CRL) is a measurement of the embryo, usually identified at 6 to 7 weeks gestation. The embryo is measured along its longest axis to obtain the CRL measurement. Crown-rump length may be used to accurately date pregnancy between 7 and 13 weeks gestation. The technique involves measurement of foetal length from the tip of the cephalic pole to the tip of caudal pole. The foetus should be at rest and assuming its natural curvature. At 5 to 6 weeks gestation, distinct landmarks cannot always be identified but easily identified from the rest of the body. After 12 weeks gestation excessive curvature of the foetus may lead to erroneous shortening of CRL measurements therefore, other measurements such as the biparietal diameter, should be used to estimate gestational age.

\section{Second and Third Trimester Assessment}

\section{Biparietal Diameter}

The biparietal diameter (BPD) is one of the most commonly measured parameters in the foetus. Campbell was the first investigator to link foetal BPD to gestational age, however, since this original report, numerous publications on this subject have appeared in the literature (Drumm et al., 1977; Hack et al., 1976; Moghissi et al., 1980). The BPD may be rapidly and reproducibly measured by ultrasound examination from 12 weeks gestation until the end of pregnancy. The BPD is imaged in the transaxial plane of the foetal head at a level depicting thalami in the midline, equidistant from the temperoparietal bones and usually the cavum septum pellucidum anteriorly. Although several methods have been used to measure BPD, the most commonly accepted method is measurement from leading edge to leading edge. 
Gestational age assignment is based on the mean BPD; however a single BPD encompasses a range of ages in which most foetuses of that size are most likely to fall.

\section{Abdominal circumference}

Measurement of the foetal abdominal circumference (AC) is obtained in the transaxial view of the foetal abdomen. The AC is measured at the level of the foetal liver, using the umbilical portion of the left portal vein as a landmark. The foetal stomach is at the same level, which is slightly caudal to the foetal heart and cephalad to the kidneys.

\section{Results and Discussion}

\section{Gravida wise distribution}

In the present study 72 cases (48\%) were primis, 50 cases $(33.33 \%)$ were gravid II and 28 cases $(18.67 \%)$ were gravid III and above (Table 1).

\section{Age wise distribution}

16 cases $(10.67 \%)$ were less than 20 years of age, 120 cases $(80 \%)$ were in $20-30$ years age group and 14 cases $(9.33 \%)$ were above 30 years (Table 2).

\section{Comparison of accuracy of BPD and FL}

The following tests of significance were applied to the above observation and the following values were obtained (Table 3 ).
' $Z$ ' test for portion -13.4 significant

Standard error of proportion - 5.0158 significant

Correlation coefficient for LMP and BPD 0.5900

Correlation coefficient for LMP and FL 0.8984

Hence FL is a better parameter.

Prediction of gestational age is an important component of obstetric sonography. Several sonographic foetal measurements have been proposed for this purpose. Most studies have used the LMP in women with regular cycles as the gold standard for true gestational age.

In the present study, gestational age calculated from LMP was taken as independent variable.

The age calculated using BPD and calculated using FL was compared with the age calculated from LMP. An amount was made to evaluate the accuracy of BPD and FL in assessing the gestational age.

Stuart Campell et al., (1970) first investigated to link the foetal BPD to the gestational age. In this study he has obtained sonar BPD measurements at each gestational age from a large number of normal gravidae in whom LMP was known and delivery occurred spontaneously within 1 week of EDD (Vintzileos et al., 1985).

Table.1 Gravida wise distribution

\begin{tabular}{|l|c|c|}
\hline Gravida & Number of cases & Percentage \\
\hline Primi & 72 & $48 \%$ \\
\hline Gravida II & 50 & $33.33 \%$ \\
\hline >= Gravida III & 28 & $18.67 \%$ \\
\hline Total & 150 & $100 \%$ \\
\hline
\end{tabular}


Table.2 Age wise distribution

\begin{tabular}{|l|c|c|}
\hline Age & Number of Cases & Percentage \\
\hline$<\mathbf{2 0}$ yrs & 16 & $10.67 \%$ \\
\hline $\mathbf{2 0 - 3 0}$ Yrs & 120 & $80 \%$ \\
\hline $\mathbf{3 0}$ Yrs & 14 & $9.33 \%$ \\
\hline
\end{tabular}

Table.3 Comparison of accuracy of BPD and FL

\begin{tabular}{|l|c|c|}
\hline Better parameter & Number of cases & Percentage \\
\hline BPD & 21 & $14 \%$ \\
\hline FL & 84 & $56 \%$ \\
\hline Both equal & 45 & $30.0 \%$ \\
\hline
\end{tabular}

Virtually all studies have demonstrated a progressive increase in variability from 20 weeks to term, but the degree to which the variability increases in the late third trimester of pregnancy have been a subject of disagreement.

Urban Waldenstron and others concluded that BPD is a better predictor of the spontaneous onset of labour than is LMP (Brenner et al., 1976).

The foetal femur length can be identified by using real time ultra sonography by the $10^{\text {th }}$ week of gestational age and normal values have been reported both as a function of menstrual age and biparietal diameter.

S. Yagel et al., compared the efficacy of FL and BPD in assessing the gestational age and concluded that FL was found to provide a more accurate index than BPD for the entire range of gestational age (Vintzileos et al., 1985).

Measurement of femur length is a more precise index of gestational than is the biparietal diameter. The use of multiple foetal growth parameters not only increases the accuracy of foetal age determination by single examination in third trimester but also gives the observer a choice to include only those parameters where technically satisfactory measurements have been obtained.

In our country where most of the women many don't keep menstrual record properly and who may present late in third trimester for the first time, ultra-sonographically assessed gestational age by multiple foetal growth parameters can be of immense value.

\section{References}

Brenner WE et al., A standard of foetal growth for the United States of America. Am J Obstet Gyneccol 126: 555, 1976.

Drumm JE et al., The prediction of delivery date by ultrasonic measurement of crown-rump length. Br. J Obstet Gynaecol 84: 1, 1977.

Hack M et al., Neonatal respiratory distress following elective delivery: A preventable disease? Am J Obstet Gynecol 126: 43, 1976.

Moghissi KS et al., Presiction and detection of Ovulation. Fertil Sterill 34: 89, 1980

Stuart Campell et al., the prediction of foetal maturity by ultrasonic measurement of the biparietal diameter. J Obstet Gynecol Br Commonw 76: 603, 1969 
Urban Waldenstron: Effects of routine one stage screening in pregnancy $-\mathrm{a}$ randomized compared trial. Lancet sept 1988.

Vintzileos AM, et al., Three fetal ponderal indexes in normal pregnancy, Obstet Gynecol 65: 807, 1985.
Yagel, S., et al., A statistical examination of the accuracy of combin FL and BPD as an index of foetal gestational age. British Journal of Obstetric and Gynaecology, 1986. Vol 93.

\section{How to cite this article:}

Farhana Aljabri, Sharmila, K.S. Saraswathi and Shyamala, R. 2018. A Hospital Based Study in South Indian Population on the Role of Ultrasound in the Estimation of Gestational Age in the Third Trimester of Pregnancy. Int.J.Curr.Microbiol.App.Sci. 7(03): 1492-1497. doi: https://doi.org/10.20546/ijcmas.2018.703.177 